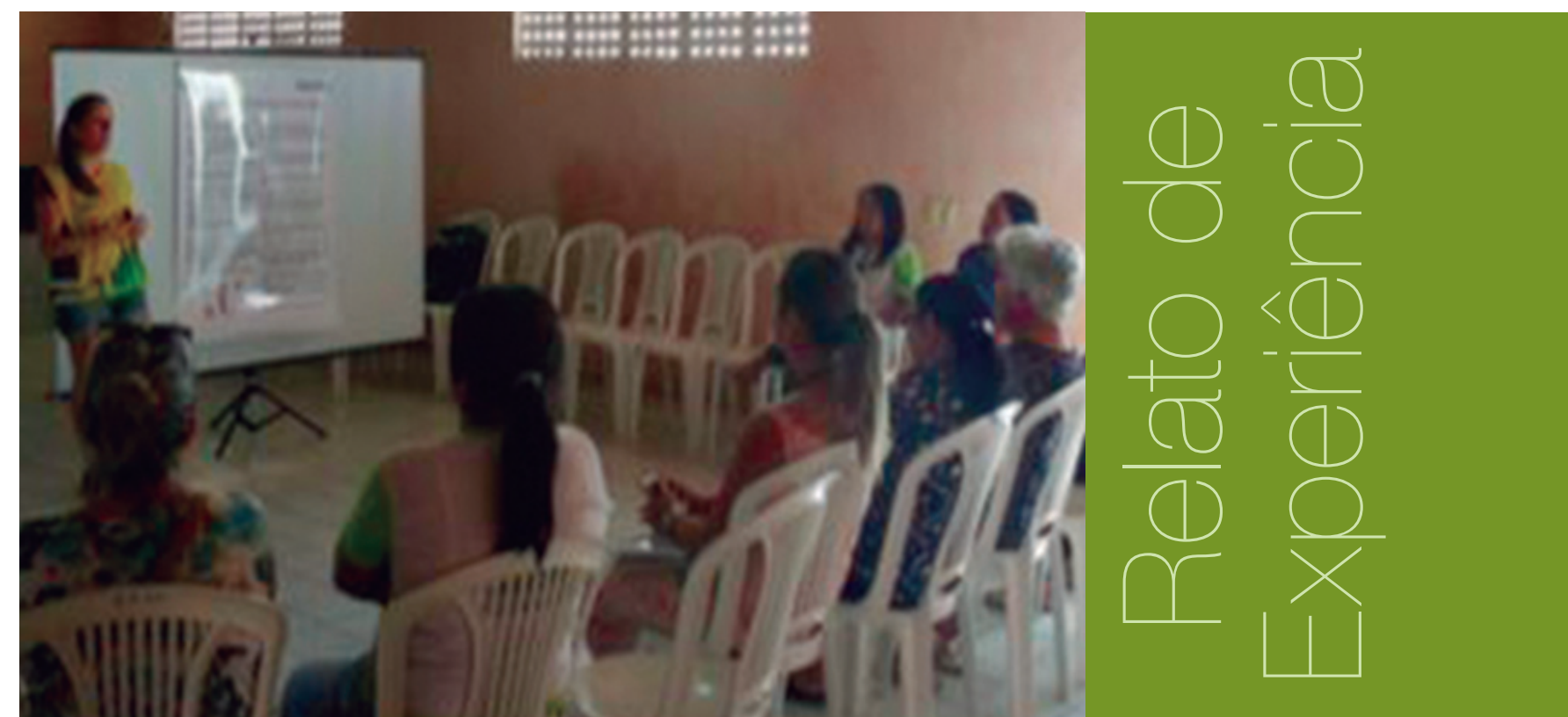

\title{
Monitoramento e rastreamento
} do câncer de útero e de mama: experiência de educação em saúde no Projeto Rondon

Giovana Cossio Rodriguez - giovanacossio@gmail.com

Sidnéia Tessmer Casarin² - stcasarin@gmail.com

Michele Mandagará de Oliveira ${ }^{3}$ - mandagara@hotmail.com

Camila do Canto Perez ${ }^{4}$ - camilacperez@gmail.com

\section{RESUMO}

Este artigo tem por objetivo relatar a experiência de extensão acadêmica acerca da promoção da saúde e prevenção do câncer de colo de útero e de mama, desenvolvida por acadêmicos e professores da Universidade Federal de Pelotas durante o Projeto Rondon Operação Mandacaru. As ações foram direcionadas aos profissionais de saúde e mulheres usuárias dos serviços de atenção básica. Foram realizadas oito oficinas, as quais atingiram diretamente noventa e duas pessoas. As oficinas proporcionaram o debate entre os participantes do projeto e a população alvo, promovendo a troca de conhecimentos e experiências para superar as dificuldades e facilitar as ações de combate ao câncer ginecológico.

\section{PALAVRAS-CHAVE}

Extensão Universitária. Projeto Rondon. Saúde da Mulher.

\footnotetext{
1 Graduada em Enfermagem pela Universidade Federal de Pelotas.

2 Professora Assistente do curso de Enfermagem da Universidade Federal de Pelotas.

3 Professora Adjunta do curso de Enfermagem da Universidade Federal de Pelotas.

4 Acadêmica do curso de Psicologia da Universidade Federal de Pelotas.
} 


\section{ABSTRACT}

This article aims to describe the academic extension experience about health promotion and cervical and breast cancer prevention developed by academics and university professors from the Universidade Federal de Pelotas during the Rondon Project - Mandacaru Operation. The actions developed were directed to health care professionals and women users of primary care services. Eight workshops were developed which reached directly ninety two people. These developed workshops promoted rich debates between the project participants and the target population, promoting the exchange of knowledge and experience to overcome difficulties and facilitate actions to combat gynecologic cancer.

\section{KEYWORDS}

University Extension. Rondon project. Women's Health.

\section{Relato de experiência}

0 Projeto Rondon é coordenado pelo Ministério da Defesa, em parceria com o Ministério da Educação e com as universidades brasileiras, e tem como objetivo auxiliar no desenvolvimento de comunidades consideradas carentes (RIEDER, 2012; SILVA; dos SANTOS, 2014). 0 projeto é desenvolvido através de operações- que são definidas como imersões, com duração média de 15 dias, nos locais previamente selecionados.

A operação Mandacaru abrangeu 15 municípios do estado do Ceará, com a participação de 30 instituições e 0 envolvimento de 299 rondonistas (BRASIL, 2016). Os participantes foram divididos entre 0 conjunto $A$, que atuou nas áreas de Cultura, Direitos Humanos e Justiça, Educação e Saúde; enquanto o conjunto B atuou na Comunicação, Tecnologia e Produção, Meio Ambiente e Trabalho. Durante a operação Mandacaru, a equipe da Universidade Federal de Pelotas (Fig. 1) integrou as ações do conjunto A.

Figura 1: Equipe de rondonistas integrantes da Operação Mandacaru, conjunto A da UFPEL.

Fonte: Acervo fotográfico do Projeto Rondon da UFPEL

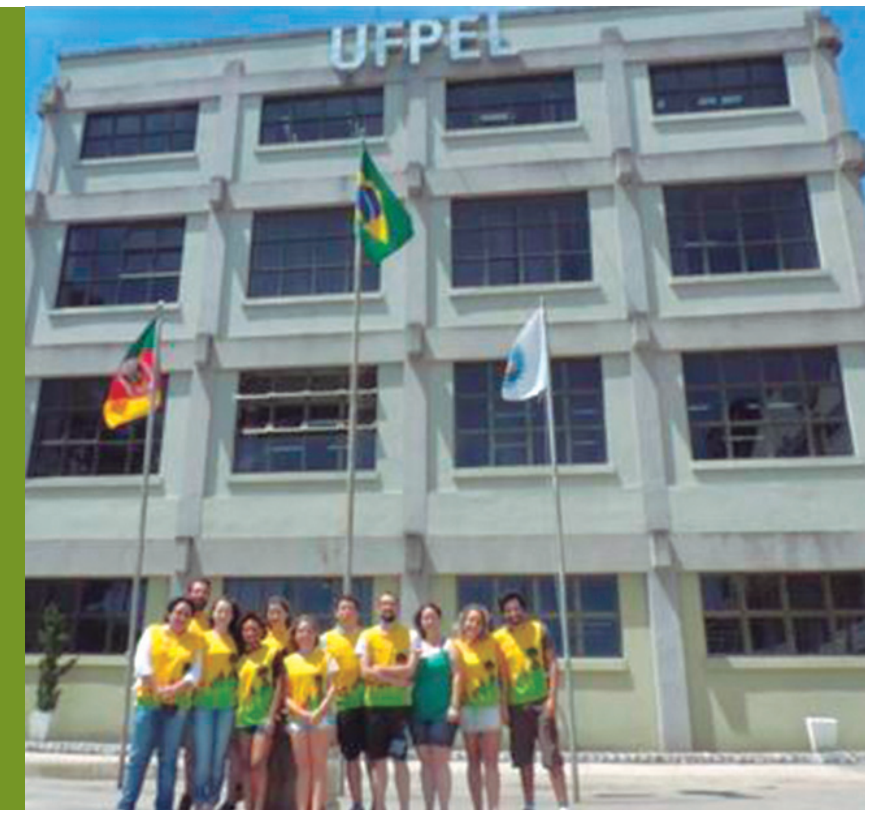

Para aplicabilidade do projeto, foram escolhidas temáticas a serem trabalhadas na comunidade com base na discussão de relevância dos temas e dados populacionais ancorados na literatura científica atual. Estima-se que por ano sejam registrados em média 500 mil novos casos de câncer de colo de útero no mundo, e que pelo menos a metade destes casos resultam em óbitos (RODRIGUES et al., 2012; BRASIL, 2013). No Brasil, estima-se que sejam registrados em média 18 mil novos casos por ano, sendo o câncer de colo de útero a terceira neoplasia que mais acomete as mulheres (MELO; SALIMENA; SOUZA, 2012). 
Tem-se como protocolo de atividade nas Unidades Básicas de Saúde (UBS) a realização de exame citopatológico e exame clínico das mamas, práticas que podem ser realizadas pelos profissionais médicos ou enfermeiros (BRASIL, 2011; 2013). Tais práticas visam a promover a saúde das mulheres e prevenir os cânceres de útero e de mama a partir do rastreamento e monitoramento de mulheres que possuem idade entre 25 e 64 anos (BRASIL, 2011; 2013).

Com isso, percebeu-se a necessidade de atenção à saúde da mulher e decidiu-se pelo trabalho com profissionais de saúde e mulheres usuárias dos serviços de atenção básica, acerca do rastreamento e do monitoramento do câncer de colo de útero e de mama.

Na prática foram realizadas atividades de educação continuada em saúde frente ao monitoramento e rastreamento do câncer de mama e de colo de útero. Porém, tendo em vista as particularidades dos grupos, foram propostas duas oficinas diferentes: uma para os profissionais de saúde, intitulada "Monitoramento das ações de prevenção do câncer de colo de útero", e outra para as mulheres usuárias dos serviços de atenção básica, intitulada "Saúde da mulher com foco no câncer ginecológico".

A oficina "Monitoramento das ações do câncer de colo de útero" foi desenvolvida pensando em sensibilizar os profissionais da atenção básica (enfermeiros, médicos, agentes comunitários de saúde, auxiliar/técnicos de enfermagem e recepcionistas) para realizar o monitoramento das ações de prevenção do câncer de colo de útero e de mama- captação das mulheres na faixa etária prioritária para os dois tipos de câncer, busca ativa, qualidade dos registros, levantamento e avaliação dos indicadores de qualidade. Para tanto, foram realizados quatro grupos de discussão em quatro UBS, sendo três rurais e uma urbana.

Para o desenvolvimento das atividades, primeiramente os profissionais de saúde foram reunidos e questionados sobre a situação da prevenção do câncer de colo de útero e mama na área de cobertura da unidade básica de saúde. Após esta etapa, os rondonistas fizeram exposiçã̃o sobre a importância da equipe estar organizada para realizar o monitoramento da ação programática e formas para facilitar a identificação das usuárias que não acessam o serviço.

Foram expostos, aos profissionais de saúde, materiais de suporte ao monitoramento, como: planilhas de acompanhamento e utilização de fichas espelho, as quais poderiam ser adotadas e/ou adaptadas para a realidade da equipe. Também foi utilizado, como recurso visual, uma apresentação em slides a respeito dos aspectos conceituais referentes ao monitoramento das ações em saúde da mulher.

Participaram das atividades cerca de 32 profissionais de saúde (médicos, dentistas, enfermeiros, agentes comunitários de saúde e técnicos de enfermagem) (Fig 2.) Esperou-se com essas atividades, a curto prazo, a promoção da reflexão sobre a importância do monitoramento das ações de prevenção do câncer ginecológico; a médio e longo prazo, espera-se a melhora nos resultados da cobertura da realização dos exames preventivos.

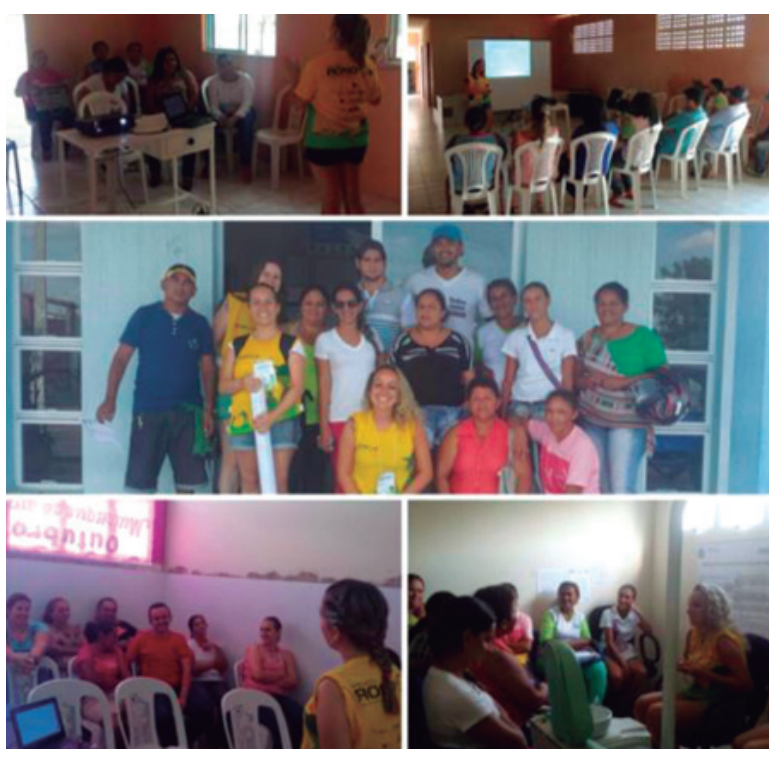

Figura 2: Mosaico com

fotos das oficinas realizadas com profissionais de saúde.

Fonte: Acervo fotográfico do

Projeto Rondon da UFPEL 
A oficina intitulada "Saúde da mulher com foco no câncer ginecológico" visou a sensibilizar a população feminina usuária dos serviços de atenção básica para a prevenção dos cânceres ginecológicos e, assim, promover 0 empoderamento das mesmas em relação à prevenção dos cânceres ginecológicos. Foram realizadas quatro atividades coletivas de educação em saúde com a comunidade em geral, sendo três em comunidades rurais e uma na área urbana do município. Participaram das quatro oficinas cerca de 60 mulheres (Fig 3).

Inicialmente, as rondonistas abordavam o tema a partir de um banner produzido previamente. Os temas abordados envolveram aspectos referentes ao câncer de mama e ao câncer de colo de útero, exames de rastreamento, sinais de alerta e formas de prevenção, chamando atenção aos fatores de risco nas mulheres entre a faixa etária mais prevalente.

Ao iniciar a atividade, as rondonistas deixavam claro que as perguntas poderiam ser realizadas a qualquer momento. Dessa forma, as atividades foram sempre dinâmicas, proporcionando a problematização do tema e não centralizando a atenção apenas nas rondonistas. Ao final das oficinas foi distribuído um folheto, contendo as informações discutidas durante as atividades.

Figura 3: Mosaico com fotos das oficinas realizadas com as mulheres usuárias

dos serviços de atenção básica de saúde.

Fonte: Acervo fotográfico do Projeto Rondon da UFPEL
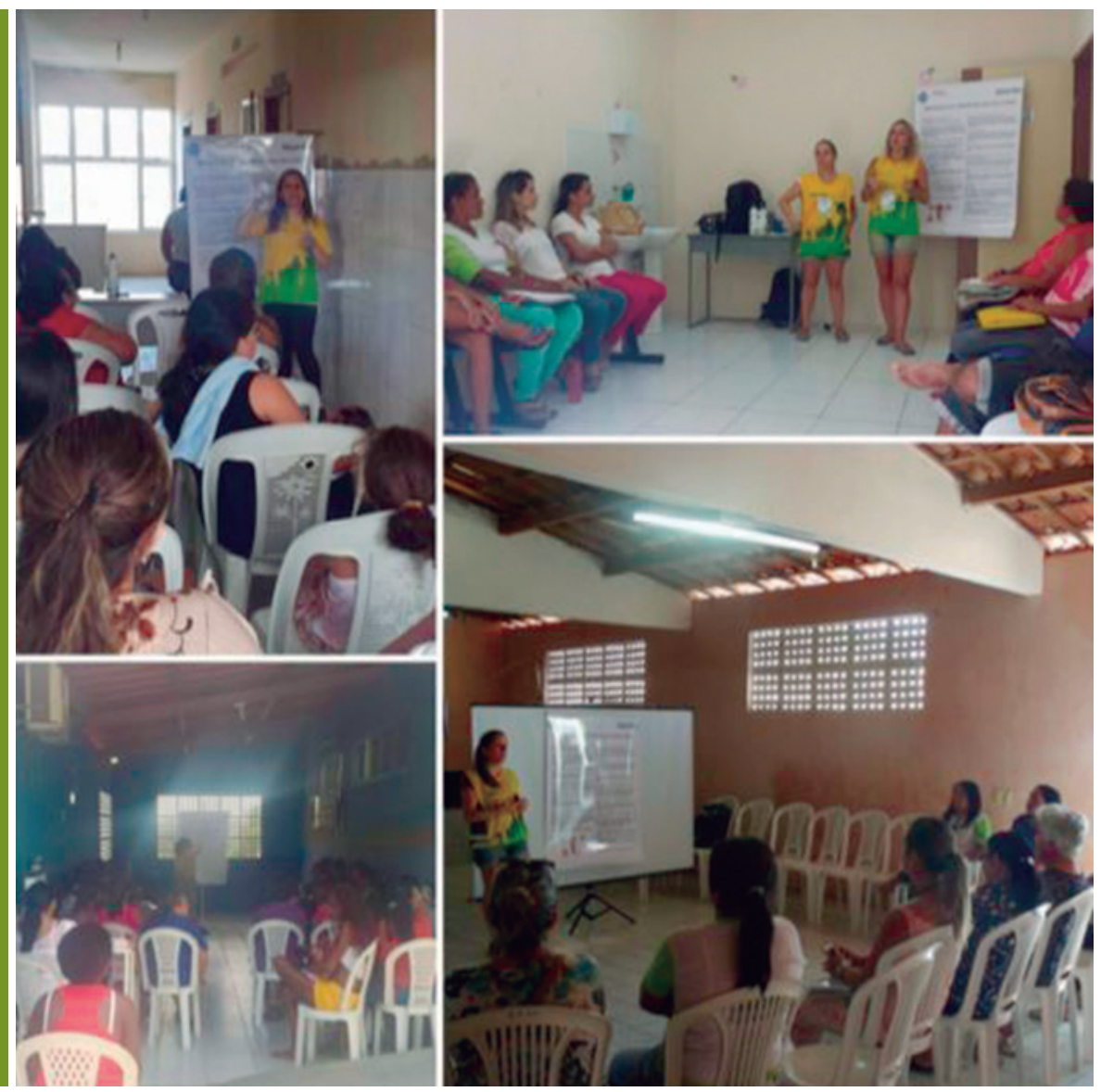

A curto prazo, essa oficina almeja 0 empoderamento e sensibilização das mulheres para realizarem os exames de prevenção do câncer de colo de útero e mama; a médio e longo prazo, a melhora na cobertura dos exames de prevenção dos cânceres ginecológicos.

As oficinas foram avaliadas pelos participantes como positivas, potenciais indutoras de outras ações e iniciativas de educação em saúde, além de serem passiveis de replicação em outras comunidades.

As principais dificuldades centraram-se em três aspectos: 0 deslocamento até as UBS, que estão localizadas distantes da área central do município; o espaço físico para as atividades, que ocorreram nas UBS, algumas sem climatização e com espaço físico inadequado (uma das oficinas precisou ocorrer no consultório odontológico); a manutenção do cronograma, que teve que ser readequado várias vezes, a fim de poder atingir um maior número de pessoas. 
As dificuldades, contudo, não superaram os pontos positivos da prática extensionista, uma vez que os participantes se mostraram interessados nos temas debatidos, participando ativamente das atividades. Além disso, é importante afirmar que as atividades de extensão desenvolvidas no Projeto Rondon possuem fundamental importância para os acadêmicos e professores, os quais podem ultrapassar as fronteiras acadêmicas do conhecimento universitário, chegando à comunidade e, junto a ela, construir um novo saber. Ressaltando também a experiência marcante na formação profissional e que certamente refletirá na vida pessoal.

\section{Referências}

BRASIL. Projeto Rondon - Institucional. Ministério da Defesa. Disponível em: <http://www. projetorondon.defesa.gov.br/portal/index/pagina/id/343/area/C/module/default>. Acesso em: 24 jul. 2015.

BRASIL. Projeto Rondon - Operações Realizadas. Ministério da Defesa. Disponível em: <http:// www. projetorondon.defesa.gov.br/portal/operacao/realizadas/module/default?id=108429> Acesso em: 05 de ago. 2016.

BRASIL. Instituto Nacional de Câncer. Diretrizes brasileiras para 0 rastreamento do câncer do colo do útero. Rio de Janeiro: INCA, 2011.

BRASIL. Ministério da Saúde. Caderno de atenção básica nº13: controle dos cânceres do colo do útero e da mama. Brasília: Editora do Ministério da Saúde, 2013.

de MELO, Maria Carmen Simões Cardoso, et al. 0 enfermeiro na prevenção do câncer do colo do útero: 0 cotidiano da atenção primária. Revista Brasileira de Cancerologia, v.5, n.3, p.389-398, 2012.

RIEDER, Arno. A extensão universitária através do Projeto Rondon: participação das universidades públicas de Mato Grosso. Revista GUAL, Florianópolis, v.5, n.2, p. 58-71, 2012.

RODRIGUES, Bruna Cortez. et al. Educação em Saúde para a Prevenção do Câncer CérvicoUterino. Revista Brasileira de Educação Médica, v. 36, n. 1, p.149-154, 2012.

SILVA, Daniele Sousa.; dos SANTOS, Jerusa Nunes Neiva Eulalio. 0 exercício da responsabilidade social e cidadania: experiência no Projeto Rondon dos estudantes da Faculdade Projeção.

Periódico Científico Outras Palavras, v.10, n.1, p.88-96, jun. 2014. 